\title{
Discovery of Novel Small Molecule FAK Activators Promoting Mucosal Healing
}

Qinggang Wang ${ }^{1 *}$, Ricardo Gallardo-Macias ${ }^{2} *$ Rashmi $^{1}$, Mikhail Y. Golovko ${ }^{3}$, Ahmed Adham Raafat Elsayed $^{1}$, Shyam K. More ${ }^{1}$, Sema Oncel ${ }^{3}$, Vadim J. Gurvich ${ }^{2}$, Marc D. Basson ${ }^{4}$.

${ }^{1}$ Department of Surgery, University of North Dakota School of Medicine and Health Sciences

${ }^{2}$ Institute for Therapeutics Discovery and Development and Department of Medicinal Chemistry, College of Pharmacy, University of Minnesota

${ }^{3}$ Department of Biomedical Sciences, University of North Dakota School of Medicine and Health Sciences

${ }^{4}$ Departments of Surgery, Biomedical Sciences, and Pathology, University of North Dakota School of Medicine and Health Sciences

Editorial correspondence should be addressed to

Dr. Marc Basson

Senior Associate Dean for Medicine and Research

University of North Dakota School of Medicine and Health Sciences

1301 North Columbia Road

Grand Forks, ND 58201

marc.basson@und.edu 


\section{TABLE OF CONTENTS}

Table S1. Predicted properties of 3, 6, 11, and $\mathbf{1 2}$ using QikProp

Table S2. Predicted properties of $\mathbf{3}, \mathbf{1 4}, \mathbf{1 5}$, and 16 using QikProp

Table S3. Purity of compounds 1-13 purchased from Enamine Ltd.

Experimental Procedures and Spectroscopic Data 
Table S1. Predicted properties of 3, 6, 11, and 12 using QikProp

\begin{tabular}{|c|c|c|c|c|}
\hline Compound ID & 3 & 6 & 11 & 12 \\
\hline MW (g/mol) & 420.434 & 466.502 & 425.493 & 419.446 \\
\hline $\begin{array}{l}\text { Aqueous } \\
\text { solubility }(\log S)\end{array}$ & -4.00 & -4.957 & $-7.458 *$ & $-6.890 *$ \\
\hline $\begin{array}{l}\text { Octanol/Water } \\
\operatorname{cog} \mathrm{P}(\log \mathrm{P})\end{array}$ & 4.202 & 4.071 & 5.319 & 5.151 \\
\hline PSA (A) & -4.00 & 66.915 & 39.608 & 41.501 \\
\hline $\begin{array}{l}\mathrm{hERG} \\
\left(\operatorname{logIC} \mathrm{C}_{50}\right)\end{array}$ & 4.202 & -5.037 & -3.637 & -4.270 \\
\hline $\begin{array}{l}\text { Serum protein } \\
\text { binding }(\log K)\end{array}$ & 58.833 & 0.333 & 0.870 & 0.721 \\
\hline $\begin{array}{l}\text { Caco-2 } \\
\text { permeability } \\
(\mathrm{mm} / \mathrm{sec})\end{array}$ & -2.656 & 691 & 6282 & 5870 \\
\hline $\begin{array}{l}\text { MDCK } \\
\text { permeability } \\
(\mathrm{mm} / \mathrm{sec})\end{array}$ & 0.209 & 2652 & 10000 & 10000 \\
\hline $\begin{array}{l}\text { Number of } \\
\text { primary } \\
\text { metabolites }\end{array}$ & 1318 & 5 & 2 & 4 \\
\hline Rotable bonds & 5161 & 6 & 1 & 1 \\
\hline $\begin{array}{l}\text { Human oral } \\
\text { absorption }\end{array}$ & 5 & high & low & low \\
\hline
\end{tabular}

* indicates a violation of the $95 \%$ range from QikProp. 
Table S2. Predicted properties of 3, 14, 15, and 16 using QikProp

\begin{tabular}{|c|c|c|c|c|}
\hline Compound ID & 3 & 14 & 15 & 16 \\
\hline MW (g/mol) & 420.434 & 456.034 & 352.435 & 388.035 \\
\hline $\begin{array}{l}\text { Aqueous } \\
\text { solubility }(\log S)\end{array}$ & -4.00 & -3.96 & -3.36 & -3.70 \\
\hline $\begin{array}{l}\text { Octanol/Water } \\
\operatorname{cog} \mathrm{P}(\log \mathrm{P})\end{array}$ & 4.202 & 3.497 & 2.860 & 2.936 \\
\hline $\operatorname{PSA}(\mathrm{A})$ & 58.833 & 58.144 & 58.269 & 57.596 \\
\hline $\begin{array}{l}\text { hERG } \\
\left(\log \mathrm{IC}_{50}\right)\end{array}$ & -2.656 & -2.646 & -2.889 & -3.192 \\
\hline $\begin{array}{l}\text { Serum protein } \\
\text { binding }(\log K)\end{array}$ & 0.209 & 0.209 & 0.027 & 0.072 \\
\hline $\begin{array}{l}\text { Caco-2 } \\
\text { permeability } \\
(\mathrm{mm} / \mathrm{sec})\end{array}$ & 1318 & 1340 & 2279 & 1986 \\
\hline $\begin{array}{l}\text { MDCK } \\
\text { permeability } \\
(\mathrm{mm} / \mathrm{sec})\end{array}$ & 5161 & 5134 & 1979 & 1709 \\
\hline $\begin{array}{l}\text { Number of } \\
\text { primary } \\
\text { metabolites }\end{array}$ & 5 & 5 & 5 & 5 \\
\hline Rotable bonds & 1 & 1 & 1 & 1 \\
\hline $\begin{array}{l}\text { Human oral } \\
\text { absorption }\end{array}$ & high & high & high & high \\
\hline
\end{tabular}


Table S3. Purity of compounds 1-13 purchased from Enamine Ltd. ${ }^{\mathrm{a}}$

\begin{tabular}{|c|c|c|c|c|c|}
\hline Compound & Exact mass & $\begin{array}{c}\text { Expected } \\
\mathbf{m} / \mathbf{z} \\
{[\mathbf{M}+\mathbf{H}]^{+}}\end{array}$ & $\begin{array}{c}\text { Observed } \\
\mathbf{m} / \mathbf{z}[\mathbf{M}+\mathbf{H}]^{+}\end{array}$ & $\begin{array}{c}\text { Mass } \\
\text { difference, ppm }\end{array}$ & Purity \% \\
\hline M1 & 343.1508 & 344.1586 & 344.1595 & 3.8 ppm & $\geq 95 \%$ \\
\hline M2 & 449.1926 & 450.2005 & 450.2005 & 0.0 ppm & $\geq 95 \%$ \\
\hline M3 & 420.1173 & 421.1851 & 421.1856 & $1.2 \mathrm{ppm}$ & $\geq 95 \%$ \\
\hline M4 & 437.2039 & 438.2117 & 438.2118 & $0.2 \mathrm{ppm}$ & $\geq 95 \%$ \\
\hline M5 & 409.1613 & 410.1692 & 410.1696 & 1.0 ppm & $\geq 95 \%$ \\
\hline M6 & 466.2192 & 467.2270 & 467.2171 & $0.2 \mathrm{ppm}$ & $\geq 93 \%$ \\
\hline M7 & 415.1663 & 416.1741 & 416.1740 & $-0.2 \mathrm{ppm}$ & $\geq 90 \%$ \\
\hline M8 & 379.2260 & 380.2338 & 380.2339 & $0.3 \mathrm{ppm}$ & $\geq 90 \%$ \\
\hline M9 & 407.1821 & 408.1899 & 408.1899 & 0.0 ppm & $\geq 95 \%$ \\
\hline M10 & 385.1977 & 386.2055 & 386.2061 & $1.6 \mathrm{ppm}$ & $\geq 95 \%$ \\
\hline M11 & 425.2290 & 426.2368 & 426.2366 & -0.5 ppm & $\geq 94 \%$ \\
\hline M12 & 419.1821 & 420.1899 & 420.1898 & $-0.2 \mathrm{ppm}$ & $\geq 95 \%$ \\
\hline M13 & 425.1385 & 426.1463 & 426.1467 & 0.9 ppm & $\geq 95 \%$ \\
\hline
\end{tabular}

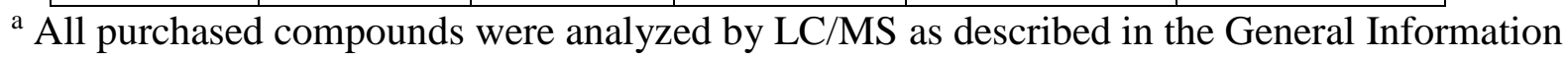
Chemistry 


\section{Experimental Procedures}

\section{Biology}

\section{FAK activation}

For studies of FAK activation in suspended Caco- 2 cells, $1 \times 10^{6}$ cells were seeded into each well of 12 -well plates pacificated to prevent adhesion. After one hour incubation at $37^{\circ} \mathrm{C}$ with either the vehicle control (DMSO unless the molecule was water-soluble) or one of the potential small molecule FAK activators, cells were lysed with 100ul of protein lysis buffer to extract protein. 30$35 \mathrm{ug}$ of protein (12-20ul of protein lysate) was loaded per lane onto SDS-PAGE gels for resolution and Western blotting.

\section{Cell culture}

Human Caco-2 cells were from ATCC (Manassas, VA) and maintained in Dulbecco's Modified Essential Eagle's Medium supplemented with 10\% FBS as described by us previously ${ }^{25} .80-90 \%$ confluent Caco-2 cells were seeded into cell culture plates pacificated with $1 \%$ heat inactivated bovine serum albumin to prevent adhesion and avoid adhesion-associated background FAK activation. Suspended cells were then treated with DMSO $(0.1 \%)$ or small molecules for 1 hour before harvesting for Western blotting.

\section{Western blotting}

Cells were lysed and protein concentration was determined via bicinchoninic acid protein assay (Thermo Fisher, Rockford, IL). The proteins were then separated by SDS-PAGE, transferred to nitrocellulose membranes, and incubated with antibodies to FAK-Tyr-397 (ab81298,1:1000 dilution), or FAK (Anti-FAK, clone 4.47, 05-537, 1:1000 dilution EMD Millipore, Temecula, 
CA). Images were taken using a LICOR -Odyssey-Fc imaging system (LI-COR Biosciences, Lincoln, NE). Densitometry was conducted on exposures within the linear range, and FAK phosphorylation was calculated as the ratio of FAK-Tyr-397 intensity to FAK intensity.

\section{Monolayer wound closure assays}

Caco-2 cells were seeded at $75-80 \%$ confluence into collagen I coated 6-well plates. When the cells reached $100 \%$ confluence, wounds were made in the monolayers with non-barrier autoclaved tips. Wound images were captured using an inverted light microscope (OLYMPUS CK2, Center Valley, PA) at 0 hours and 24 hours after treatment with DMSO or small molecules. Wound areas were measured with Image J software.

\section{Statistical analysis}

Data are depicted graphically as mean +/- standard error, and were analyzed by t-test or ANOVA as appropriate seeking $95 \%$ confidence.

\section{Method}

C57Bl/6J mice were given a single intraperitoneal injection of $900 \mathrm{ug} / \mathrm{kg}$ dose of compound 3. Mice were anesthetized and blood drawn by cardiac puncture for plasma measurements of compound 3 levels at 0-12 hours after dosing, and compound 3 plasma concentrations were measured by LC-MS. In a separate set of mice, ischemic jejunal ulcers were created as previously described ${ }^{1,2}$ by placing $3.14 \mathrm{~mm}^{2}$ filter paper disks soaked in $75 \%$ acetic acid on the serosa of the jejunum for 15 seconds at laparotomy. 24 hours later, animals were randomly assigned to receive either compound 3 at $900 \mathrm{ug} / \mathrm{kg}$ or DMSO vehicle control by intraperitoneal injection every six hours for three days. At day four, mice were anesthetized, blood was drawn by cardiac puncture, and animals were sacrificed by cervical dislocation before removing the jejunal ulcer segment. 
Ischemic ulcers were imaged with an OLYMPUS Q Color 5 digital camera (Center Valley, PA); ulcer area was measured with Image J software (NIH). All of these studies were approved by the IACUC of the University of North Dakota.

\section{General Information}

\section{Chemistry}

All reagents were purchased from commercial suppliers and used without further purification. Compounds 1-13 were purchased from Enamine Ltd with $>90 \%$ purity. All solvents used were reagent quality grade or anhydrous solvents. All reactions were performed under an inert atmosphere of nitrogen gas unless noted otherwise. ${ }^{1} \mathrm{H}$ NMR spectra were recorded on a Bruker Advance II (400 MHz). ${ }^{1} \mathrm{H}$ NMR data are reported as follows: chemical shift [multiplicity (s = singlet, $\mathrm{d}=$ doublet, $\mathrm{t}=$ triplet, $\mathrm{q}=$ quartet, $\mathrm{dd}=$ doublet of doublets, $\mathrm{m}=$ multiplet, $\mathrm{s} \mathrm{br}=$ broad singlet), $\mathrm{J}=$ coupling constant(s) $(\mathrm{Hz})$, integration]. Column chromatography was carried out employing Siliaflash silica gel (P60, 63-200 um). Precoated silica gel plates F-254 were used for thin-layer analytical chromatography. All final compounds were purified to $>95 \%$ purity as determined by LC/MS. Compounds were analyzed using a quadrupole/time of flight MS (G2S, Waters) coupled to UPLC binary pump system (Acquity, Waters). Compounds were resolved on ACQUITY UPLC HSS T3 column $(1.8 \mu \mathrm{M}, 100 \AA \AA$ pore diameter, $2.1 \times 150 \mathrm{~mm}$; Waters $)$ with a gradient of $0.1 \%$ formic acid in water (Solvent A) and acetonitrile with $0.1 \%$ formic acid (solvent B) at $0.3 \mathrm{~mL} / \mathrm{min}$. Percent B was increased from $1 \%$ to $75 \%$ over 2 min and then to $99 \%$ over 3 min. At 6 min, \%B was returned back to $1 \%$ over 1 min. Data were collected with $\mathrm{MS}^{\mathrm{E}}$ method, 
and MS was operated in sensitivity mode. Compounds were ionized with a positive electrospray ionization technique.

\section{Synthesis of Compounds 6, 12, and 14-23, 25, 27-29. ${ }^{3}$}

Synthesis of Phenyl (2-morpholino-5-(trifluoromethyl)phenyl)carbamate (22)

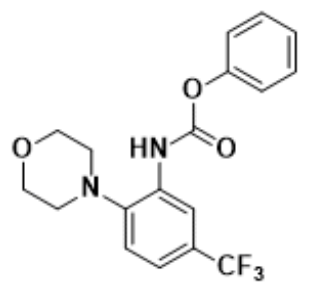

22

To a stirred solution of 2-morpholino-5-trifluoromethyl aniline (0.8 g, $3.2 \mathrm{mmol})$ in dry DCM (25 $\mathrm{mL})$, under $\mathrm{N}_{2}$ atmosphere at $0^{\circ} \mathrm{C}$, was added a solution of phenyl chloroformate $(0.6 \mathrm{~mL}, 4.8$ $\mathrm{mmol})$ in dry DCM $(5 \mathrm{~mL})$, followed by pyridine $(0.65 \mathrm{~mL}, 8.10 \mathrm{mmol})$. The crude reaction was allowed to warm up at room temperature and stirred overnight. Crude reaction was concentrated in vacuo and product was purified by column chromatography using hexane: EtOAc (70:30) to afford pure product as a clear oil in $85 \%$ yield. ${ }^{1} \mathrm{H}$ NMR $\left(\mathrm{CDCl}_{3}, 400 \mathrm{MHZ}\right): \delta 8.47(\mathrm{~s}, 1 \mathrm{H}), 8.19$ (s, 1H), $7.38(\mathrm{~d}, J=8.0 \mathrm{~Hz}, 2 \mathrm{H}), 7.32(\mathrm{~d}, J=8.0 \mathrm{~Hz}, 1 \mathrm{H}), 7.24(\mathrm{~d}, J=4.0 \mathrm{~Hz}, 2 \mathrm{H}), 7.19(\mathrm{~d}, J=$ $8.0 \mathrm{~Hz}, 2 \mathrm{H}), 3.91(\mathrm{t}, J=4.0 \mathrm{~Hz}, 4 \mathrm{H}), 2.92(\mathrm{t}, J=4.0 \mathrm{~Hz}, 4 \mathrm{H})$. MS (ESI+) calculated for $\mathrm{C}_{18} \mathrm{H}_{17} \mathrm{~F}_{3} \mathrm{~N}_{2} \mathrm{O}_{3}[\mathrm{M}+1]+:$ 367.3, observed: 367.3

\section{Synthesis of Phenyl (2-morpholinophenyl)carbamate (23)}

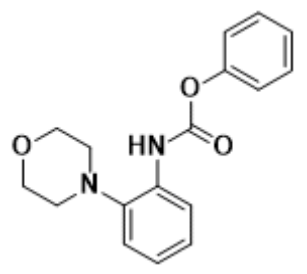


Following general procedure for the synthesis of 22, Phenyl (2-morpholinophenyl)carbamate was obtained as white solid in $80 \%$ yield. ${ }^{1} \mathrm{H}$ NMR $\left(\mathrm{CDCl}_{3}, 400 \mathrm{MHZ}\right): \delta 8.28(\mathrm{~s}, 1 \mathrm{H}), 8.16(\mathrm{~d}, \mathrm{~J}=8.0$ $\mathrm{Hz}, 1 \mathrm{H}), 7.41(\mathrm{t}, J=8.0 \mathrm{~Hz}, 2 \mathrm{H}), 7.28(\mathrm{~d}, \mathrm{~J}=8.0 \mathrm{~Hz}, 1 \mathrm{H}), 7.24(\mathrm{~d}, J=8.0 \mathrm{~Hz}, 2 \mathrm{H}), 7.20(\mathrm{~d}, J=$ $8.0 \mathrm{~Hz}, 2 \mathrm{H}), 7.08(\mathrm{t}, \mathrm{J}=8.0 \mathrm{~Hz}, 1 \mathrm{H}), 3.91(\mathrm{t}, \mathrm{J}=4.0 \mathrm{~Hz}, 4 \mathrm{H}), 2.92(\mathrm{t}, \mathrm{J}=4.0 \mathrm{~Hz}, 4 \mathrm{H})$. MS (ESI+) calculated for $\mathrm{C}_{17} \mathrm{H}_{18} \mathrm{~N}_{2} \mathrm{O}_{3}[\mathrm{M}+1]+:$ 299.3, observed: 299.3.

\section{Synthesis of 2,6-dimethyl-4-(2-nitro-4-(trifluoromethyl)phenyl)morpholine (25a)}

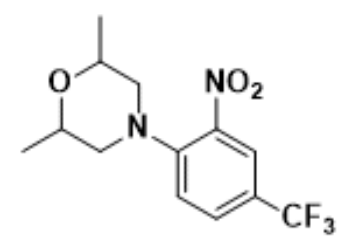

25a

To a stirred solution of 4-Fluoro-3-nitrobenzotrifluoride (1.0 g, $4.7 \mathrm{mmol})$ in dry DMF (10 mL) was added 2,6-dimethylmorpholine ( $0.6 \mathrm{~g}, 5.2 \mathrm{mmol})$, followed by $\mathrm{K}_{2} \mathrm{CO}_{3}(0.8 \mathrm{~g}, 6.2 \mathrm{mmol})$, The crude reaction was stirred under heating at $60^{\circ} \mathrm{C}$ overnight. After cooling at room temperature, crude reaction was diluted with DCM and washed with brine several times. The organic layer was dried over anhydrous $\mathrm{Na}_{2} \mathrm{SO}_{4}$, filtered, and concentrated in vacuo. Product was purified by column chromatography using hexane: EtOAc (50:50) to afford a yellow solid 76\% yield. ${ }^{1} \mathrm{H}$ NMR $\left(\mathrm{CDCl}_{3}, 400 \mathrm{MHZ}\right): \delta 8.07(\mathrm{~d}, J=4.0 \mathrm{~Hz}, 1 \mathrm{H}), 7.66(\mathrm{dd}, J=8.0 \mathrm{~Hz}, 4.0 \mathrm{~Hz}, 1 \mathrm{H}), 7.14(\mathrm{~d}, J=8.0$ $\mathrm{Hz}, 1 \mathrm{H}), 3.89-3.81(\mathrm{~m}, 2 \mathrm{H}), 3.12(\mathrm{~d}, J=4.0 \mathrm{~Hz}, 2 \mathrm{H}), 2.70(\mathrm{t}, J=12.0 \mathrm{~Hz}, 2 \mathrm{H}), 1.21(\mathrm{~d}, J=4.0$ Hz, $6 \mathrm{H})$. MS (ESI+) calculated for $\mathrm{C}_{13} \mathrm{H}_{15} \mathrm{~F}_{3} \mathrm{~N}_{2} \mathrm{O}_{3}[\mathrm{M}+1]+:$ 305.2, observed: 305 . 


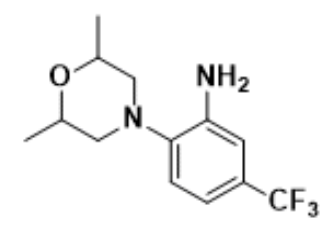

To a stirred solution of 2,6-dimethyl-4-(2-nitro-4-(trifluoromethyl)phenyl)morpholine (1.1 g, 3.6 $\mathrm{mmol})$ in $\mathrm{MeOH}(15 \mathrm{~mL})$, purged under nitrogen, was added $\mathrm{Pd} / \mathrm{C}(0.1 \mathrm{~g})$. Then, a $\mathrm{H}_{2}$ ballon was attached to the reaction mixture and stirred at room temperature for $3 \mathrm{hrs}$. Crude was filtered and concentrated in vacuo. Product was purified by column chromatography using hexane: EtOAc (20:80) to afford a clear oil $70 \%$ yield. ${ }^{1} \mathrm{H} \mathrm{NMR}\left(\mathrm{CDCl}_{3}, 400 \mathrm{MHZ}\right): \delta 7.50(\mathrm{~s}, 2 \mathrm{H}), 7.18(\mathrm{~d}, J=$ 8.0 Hz, 1H), 7.02-6.99 (m, 2H), 3.86-3.77 (m, 2H), $2.96(\mathrm{~d}, J=4.0 \mathrm{~Hz}, 2 \mathrm{H}), 2.40(\mathrm{t}, J=8.0 \mathrm{~Hz}$, $2 \mathrm{H}), 1.21(\mathrm{~d}, J=4.0 \mathrm{~Hz}, 6 \mathrm{H})$. MS (ESI+) calculated for $\mathrm{C}_{13} \mathrm{H}_{17} \mathrm{~F}_{3} \mathrm{~N}_{2} \mathrm{O}[\mathrm{M}+1]+:$ 275.2, observed: 275.2 .

Synthesis of phenyl (2-(2,6-dimethylmorpholino)-5-(trifluoromethyl)phenyl)carbamate (28)

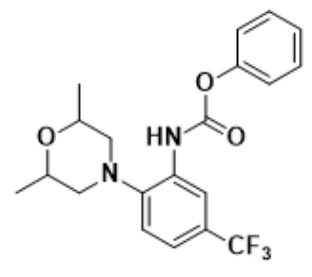

28

Following general procedure for the synthesis of 22, phenyl (2-(2,6-dimethylmorpholino)-5(trifluoromethyl)phenyl)carbamate was obtained as clear oil in $80 \%$ yield. ${ }^{1} \mathrm{H} \mathrm{NMR}\left(\mathrm{CD}_{3} \mathrm{CN}, 400\right.$ MHZ): $\delta 8.20(\mathrm{~s}, 1 \mathrm{H}), 7.42(\mathrm{t}, \mathrm{J}=8.0 \mathrm{~Hz}, 2 \mathrm{H}), 7.29-7.18(\mathrm{~m}, 5 \mathrm{H}), 3.5-3.91(\mathrm{~m}, 2 \mathrm{H}), 2.86(\mathrm{~d}, J=$ 
$12.0 \mathrm{~Hz}, 2 \mathrm{H}), 2.54(\mathrm{t}, J=12.0 \mathrm{~Hz}, 2 \mathrm{H}), 1.26(\mathrm{~d}, \mathrm{~J}=8.0 \mathrm{~Hz}, 6 \mathrm{H}) . \mathrm{MS}$ (ESI+) calculated for $\mathrm{C}_{20} \mathrm{H}_{21} \mathrm{~F}_{3} \mathrm{~N}_{2} \mathrm{O}_{3}[\mathrm{M}+1]+:$ 395.4, observed: 395.4

Synthesis of phenyl (2-(1,1-dioxidothiomorpholino)-5-(trifluoromethyl)phenyl)carbamate (29)

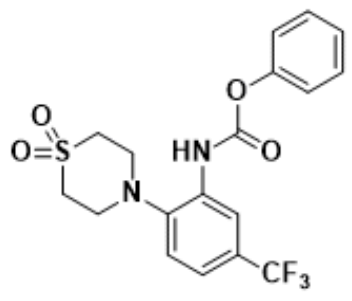

29

Following general procedure for the synthesis of 22, phenyl (2-(1,1-dioxidothiomorpholino)-5(trifluoromethyl)phenyl)carbamatewas obtained as off-white solid in $70 \%$ yield. ${ }^{1} \mathrm{H}$ NMR (Acetone- $\left.\mathrm{d}_{6}, 400 \mathrm{MHZ}\right): \delta 8.82(\mathrm{~s}, 1 \mathrm{H}), 8.41(\mathrm{~d}, \mathrm{~J}=4.0 \mathrm{~Hz}, 1 \mathrm{H}), 7.59(\mathrm{~d}, J=8.0 \mathrm{~Hz}, 1 \mathrm{H}), 7.46-$ $7.41(\mathrm{~m}, 3 \mathrm{H}), 7.29-7.23(\mathrm{~m}, 3 \mathrm{H}), 3.54(\mathrm{t}, J=4.0 \mathrm{~Hz}, 4 \mathrm{H}), 3.43(\mathrm{t}, J=4.0 \mathrm{~Hz}, 4 \mathrm{H}) . \mathrm{MS}$ (ESI+) calculated for $\mathrm{C}_{18} \mathrm{H}_{17} \mathrm{~F}_{3} \mathrm{~N}_{2} \mathrm{O}_{4} \mathrm{~S}[\mathrm{M}+1]+:$ 415.4, observed: 415 .

Synthesis of phenyl (2-(4-methylpiperazin-1-yl)-5-(trifluoromethyl)phenyl)carbamate (27)

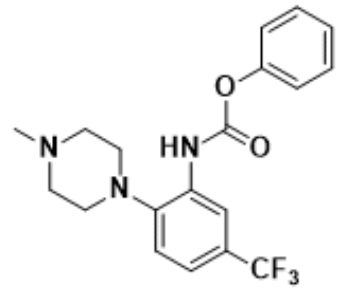


Following general procedure for the synthesis of 22, phenyl (2-(4-methylpiperazin-1-yl)-5(trifluoromethyl)phenyl)carbamate was obtained as white solid in $50 \%$ yield. ${ }^{1} \mathrm{H}$ NMR $\left(\mathrm{CDCl}_{3}\right.$, $400 \mathrm{MHZ}): \delta 8.49$ (s, 1H), 8.07 (s, 1H), $7.43(\mathrm{t}, J=8.0 \mathrm{~Hz}, 2 \mathrm{H}), 7.37$ (s, 1H), 7.30-7.26 (m, 2H), $7.21(\mathrm{~d}, J=8.0 \mathrm{~Hz}, 2 \mathrm{H}), 3.20(\mathrm{~s}, \mathrm{br}, 4 \mathrm{H}), 3.04$ (s, br, 4H), 2.66 (s, 3H). MS (ESI+) calculated for $\mathrm{C}_{19} \mathrm{H}_{20} \mathrm{~F}_{3} \mathrm{~N}_{3} \mathrm{O}_{2}[\mathrm{M}+1]+: 380.4$, observed: 380 .

Synthesis of $\quad N$-(2-morpholino-5-(trifluoromethyl)phenyl)-2-phenylpyrrolidine-1carboxamide (12).

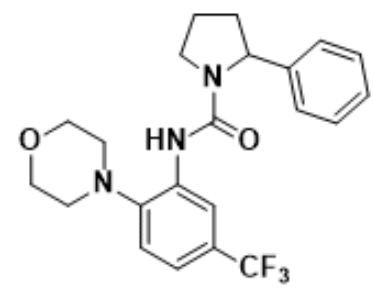

12

To a stirred solution of phenyl (2-morpholino-5-(trifluoromethyl)phenyl)carbamate (0.51 g, 1.39 $\mathrm{mmol})$ in dry pyridine ( $5 \mathrm{~mL})$, under $\mathrm{N}_{2}$ atmosphere, was added 2-phenylpyrrolidine ( $0.20 \mathrm{~g}, 1.39$ mmol). Then, the solution was heated at $80^{\circ} \mathrm{C}$ for $16 \mathrm{hrs}$. After crude was cooled down at room temperature, crude was dissolved in DCM and concentrated in vacuo. Product was purified by column chromatography using hexane: EtOAc (70:30-> 50:50) to afford pure product as a white solid in $60 \%$ yield. ${ }^{1} \mathrm{H}$ NMR $\left(\mathrm{CDCl}_{3}, 400 \mathrm{MHZ}\right): \delta 8.68(\mathrm{~s}, 1 \mathrm{H}), 7.72(\mathrm{~s}, 1 \mathrm{H}), 7.38(\mathrm{~d}, J=8.0 \mathrm{~Hz}$, 2H), 7.32-7.28 (m, 3H), $7.18(\mathrm{~d}, J=8.0 \mathrm{~Hz}, 1 \mathrm{H}), 7.12(\mathrm{~d}, J=8.0 \mathrm{~Hz}, 1 \mathrm{H}), 5.02(\mathrm{~d}, J=8.0 \mathrm{~Hz}$, 1H), 3.85-3.73 (m, 2H), $3.48(\mathrm{~s}, \mathrm{br}, 2 \mathrm{H}), 3.28(\mathrm{~s}, \mathrm{br}, 2 \mathrm{H}), 2.68-2.61(\mathrm{~m}, 4 \mathrm{H}), 2.50-2.41(\mathrm{~m}, 1 \mathrm{H})$, 2.04-1.93 (m, 3H). MS (ESI+) calculated for $\mathrm{C}_{22} \mathrm{H}_{24} \mathrm{~F}_{3} \mathrm{~N}_{3} \mathrm{O}_{2}[\mathrm{M}+1]+:$ 420.24, observed: 420.25 . 
The following compounds were synthesized following general procedure for the synthesis of $\mathbf{1 2}$

\section{1-(2-(dimethylamino)-2-(4-methoxyphenyl)ethyl)-3-(2-morpholino-5-}

(trifluoromethyl)phenyl)urea (6)

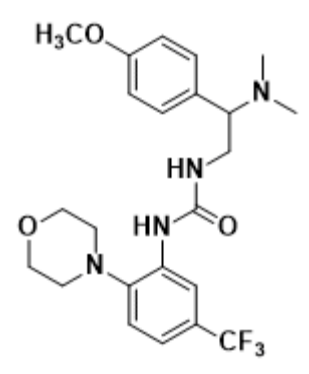

6

Obtained as a white solid in $50 \%$ yield. ${ }^{1} \mathrm{H}$ NMR $\left(\mathrm{CDCl}_{3}, 400 \mathrm{MHZ}\right): \delta 8.42(\mathrm{~s}, 1 \mathrm{H}), 7.63$ (s, br, 1H), 7.25-7.18 (m, 4H), $6.94(\mathrm{~d}, J=8.0 \mathrm{~Hz}, 2 \mathrm{H}), 6.16(\mathrm{~s}, \mathrm{br}, 1 \mathrm{H}), 3.93-3.92(\mathrm{~m}, 4 \mathrm{H}), 3.89-3.85$ (m, 2H), $3.84(\mathrm{~s}, 3 \mathrm{H}), 3.67-3.63(\mathrm{~m}, 1 \mathrm{H}), 2.90(\mathrm{t}, J=4.0 \mathrm{~Hz}, 4 \mathrm{H}), 2.39(\mathrm{~s}, 6 \mathrm{H})$. MS (ESI+) calculated for $\mathrm{C}_{23} \mathrm{H}_{29} \mathrm{~F}_{3} \mathrm{~N}_{4} \mathrm{O}_{3}[\mathrm{M}+1]+:$ 467.50, observed: 467.32.

1-(2-(dimethylamino)-2-(phenylethyl)-3-(2-morpholino-5-(trifluoromethyl)phenyl)urea (17)

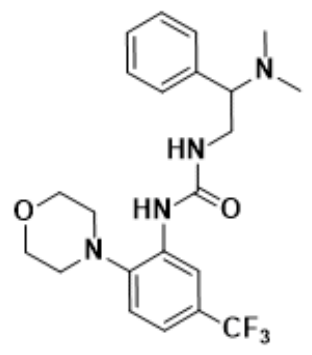

17

Obtained as a white solid in $60 \%$ yield. ${ }^{1} \mathrm{H} \mathrm{NMR}\left(\mathrm{CDCl}_{3}, 400 \mathrm{MHZ}\right): \delta 8.21(\mathrm{~s}, 1 \mathrm{H}), 7.29-7.24$ (m, 3H), 7.20-7.15 (m, 5H), $7.07(\mathrm{~d}, J=8.0 \mathrm{~Hz}, 1 \mathrm{H}), 5.16$ (s, br, 1H), 3.75-3.69 (m, 5H), 3.47- 
$3.41(\mathrm{~m}, 2 \mathrm{H}), 2.77(\mathrm{~s}, 4 \mathrm{H}), 2.16(\mathrm{~s}, 6 \mathrm{H})$. MS (ESI+) calculated for $\mathrm{C}_{22} \mathrm{H}_{27} \mathrm{~F}_{3} \mathrm{~N}_{4} \mathrm{O}_{2}[\mathrm{M}+1]+:$ 437.50, observed:437.29.

\section{2-(4-hydroxyphenyl)-N-(2-morpholino-5-(trifluoromethyl)phenyl)pyrrolidine-1-} carboxamide (18)

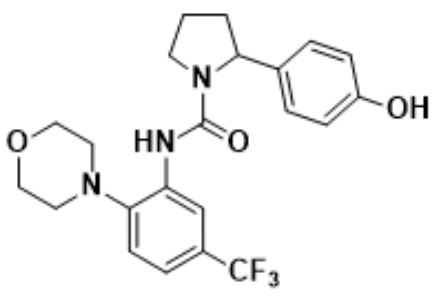

18

Obtained as a yellow solid in 60\% yield. ${ }^{1} \mathrm{H}$ NMR (DMSO- $\mathrm{d}_{6}, 400 \mathrm{MHZ}$ ): $\delta 9.36$ (s, 1H), 8.54 (s, 1H), $7.73(\mathrm{~s}, 1 \mathrm{H}), 7.34(\mathrm{~d}, J=8.0 \mathrm{~Hz}, 1 \mathrm{H}), 7.24(\mathrm{~d}, J=8.0 \mathrm{~Hz}, 1 \mathrm{H}), 7.09(\mathrm{~d}, J=8.0 \mathrm{~Hz}, 2 \mathrm{H}), 6.75$ (d, $J=8.0 \mathrm{~Hz}, 2 \mathrm{H}), 4.98(\mathrm{~d}, J=8.0 \mathrm{~Hz}, 1 \mathrm{H}), 3.69(\mathrm{~s}, 1 \mathrm{H}), 3.61-3.55(\mathrm{~m}, 3 \mathrm{H}), 3.43-3.41(\mathrm{~m}, 2 \mathrm{H})$, $2.70(\mathrm{~s}, 2 \mathrm{H}), 2.59$ (s, 2H), $2.31(\mathrm{~d}, \mathrm{~J}=8.0 \mathrm{~Hz}, 1 \mathrm{H}), 1.89-1.87(\mathrm{~m}, 1 \mathrm{H}), 1.77-1.75(\mathrm{~m}, 2 \mathrm{H}) . \mathrm{MS}$ (ESI+) calculated for $\mathrm{C}_{22} \mathrm{H}_{24} \mathrm{~F}_{3} \mathrm{~N}_{4} \mathrm{O}_{3}[\mathrm{M}+1]+:$ 435.4, observed: 436.4.

\section{N-(2-morpholinophenyl)-2-(pyridine-4-yl)pyrrolidine-1-carbxamide (15)}

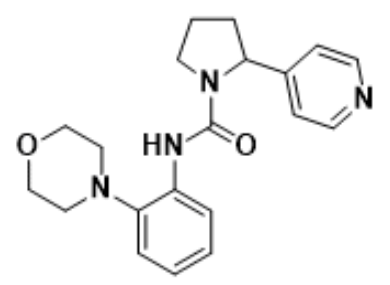

15

Obtained as a white solid in $60 \%$ yield. ${ }^{1} \mathrm{H} \mathrm{NMR}\left(\mathrm{CDCl}_{3}, 400 \mathrm{MHZ}\right): \delta 8.58(\mathrm{~d}, J=4.0 \mathrm{~Hz}, 2 \mathrm{H})$, $8.29(\mathrm{dd}, J=8.0,4.0 \mathrm{~Hz}, 1 \mathrm{H}), 7.99(\mathrm{~s}, 1 \mathrm{H}), 7.22(\mathrm{~d}, J=4.0 \mathrm{~Hz}, 2 \mathrm{H}), 7.14-7.10(\mathrm{~m}, 2 \mathrm{H}), 6.95(\mathrm{td}$, 
$J=8.0,4.0 \mathrm{~Hz}, 1 \mathrm{H}), 5.08(\mathrm{~d}, J=8.0 \mathrm{~Hz}, 1 \mathrm{H}), 3.82-3.3 .78(\mathrm{~m}, 1 \mathrm{H}), 3.74-3.68(\mathrm{~m}, 4 \mathrm{H}), 3.58(\mathrm{~s}$, 1H), 2.81-2.75 (m, 4H), 2.49-2.40 (m, 1H), 2.08-2.04 (m, 1H), 2.03-1.96 (m, 2H). MS (ESI+) calculated for C20H24F3N4O2 [M+1]+: 352.4 observed: 353.4 .

$N$-(2-(4-methylpiperzin-1-yl)-5-(trifluoromethyl)phenyl)-2-(pyridin-4-yl)pyrrolidine-1carboxamide (19)

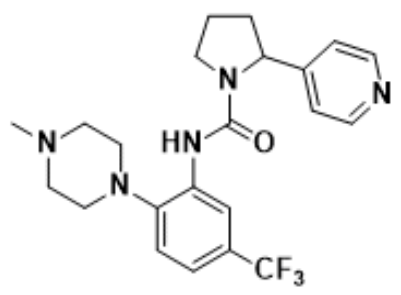

19

Obtained as a yellow semi-solid in $50 \%$ yield. ${ }^{1} \mathrm{H}$ NMR $\left(\mathrm{CD}_{3} \mathrm{OD}, 400 \mathrm{MHZ}\right): \delta 8.78$ (d, $J=8.0$ $\mathrm{Hz}, 2 \mathrm{H}), 8.31(\mathrm{~d}, J=4.0 \mathrm{~Hz}, 1 \mathrm{H}), 8.04(\mathrm{~d}, J=4.0 \mathrm{~Hz}, 2 \mathrm{H}), 7.44(\mathrm{~d}, J=8.0 \mathrm{~Hz}, 1 \mathrm{H}), 7.35(\mathrm{~d}, J=$ $8.0 \mathrm{~Hz}, 1 \mathrm{H}), 5.32-5.29(\mathrm{~m}, 1 \mathrm{H}), 4.05-4.00(\mathrm{~m}, 1 \mathrm{H}), 3.82(\mathrm{q}, J=8.0 \mathrm{~Hz}, 1 \mathrm{H}), 3.85-3.79(\mathrm{~m}, 2 \mathrm{H})$, 3.42-3.18 (m, 6H), $3.04(\mathrm{~s}, 3 \mathrm{H}), 2.66-2.57(\mathrm{~m}, 1 \mathrm{H}), 2.27-2.11(\mathrm{~m}, 2 \mathrm{H}), 2.00-1.95(\mathrm{~m}, 1 \mathrm{H}) . \mathrm{MS}$ (ESI+) calculated for $\mathrm{C}_{22} \mathrm{H}_{26} \mathrm{~F}_{3} \mathrm{~N}_{5} \mathrm{O}_{3}[\mathrm{M}+1]+:$ 433.4, observed: 434.4.

$N$-(2-(2,6-dimethylmorpholino)-5-(trifluoromethyl)phenyl)-2-(pyridin-4-yl)pyrrolidine-1carboxamide (20)

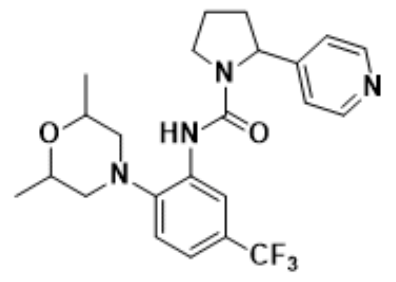


Obtained as a white semi-solid in $50 \%$ yield. ${ }^{1} \mathrm{H}$ NMR (Acetone- $\mathrm{d}_{6}, 400 \mathrm{MHZ}$ ): $\delta 8.92$ (d, $J=4.0$ $\mathrm{Hz}, 2 \mathrm{H}), 8.63(\mathrm{~d}, J=2.0 \mathrm{~Hz}, 1 \mathrm{H}), 8.07(\mathrm{~d}, J=8.0 \mathrm{~Hz}, 2 \mathrm{H}), 7.42(\mathrm{~d}, J=8.0 \mathrm{~Hz}, 1 \mathrm{H}), 7.29(\mathrm{~d}, J=$ 8.0 Hz, 1H), 5.40-5.37 (m, 1H), 4.09-4.05 (m, 1H), 3.92-3.79 (m, 3H), $2.91(\mathrm{~d}, J=8.0 \mathrm{~Hz}, 2 \mathrm{H})$, 2.67-2.52 (m, 2H), $2.42(\mathrm{t}, J=12.0 \mathrm{~Hz}, 1 \mathrm{H}), 2.23-2.18(\mathrm{~m}, 2 \mathrm{H}), 1.91-188(\mathrm{~m}, 1 \mathrm{H}), 1.18(\mathrm{~d}, J=4.0$ $\mathrm{Hz}, 3 \mathrm{H}), 1.15(\mathrm{~d}, J=4.0 \mathrm{~Hz}, 3 \mathrm{H})$. MS (ESI+) calculated for $\mathrm{C}_{23} \mathrm{H}_{27} \mathrm{~F}_{3} \mathrm{~N}_{4} \mathrm{O}_{2}[\mathrm{M}+1]+:$ 448.5, observed: 449.5 .

$N$-(2-(1,1-dioxidothiomorpholino)-5-(trifluoromethyl)phenyl)-2-(pyridin-4-yl)pyrrolidine-1carboxamide (21)

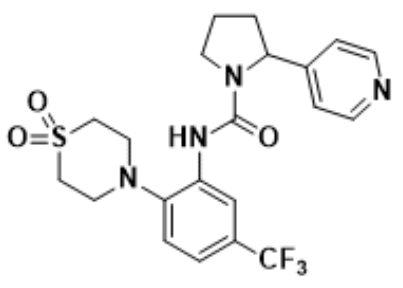

21

Obtained as a white solid in $50 \%$ yield. ${ }^{1} \mathrm{H}$ NMR (Acetone- $\mathrm{d}_{6}, 400 \mathrm{MHZ}$ ): $\delta 8.87$ (d, $J=8.0 \mathrm{~Hz}$, 2H), $8.63(\mathrm{~s}, 1 \mathrm{H}), 7.99(\mathrm{~d}, J=8.0 \mathrm{~Hz}, 2 \mathrm{H}), 7.58(\mathrm{~d}, J=8.0 \mathrm{~Hz}, 1 \mathrm{H}), 7.32(\mathrm{dd}, J=4.0 \mathrm{~Hz}, 2.0 \mathrm{~Hz}$, 1H), 5.41-5.38 (m, 1H), 4.21-4.16 (m, 1H), $3.97(\mathrm{q}, J=8.0 \mathrm{~Hz}, 1 \mathrm{H}), 3.46-3.43(\mathrm{~m}, 4 \mathrm{H}), 3.36-3.33$ (m, 4H), 2.65-2.56 (m, 1H), 2.24-2.12 (m, 3H), 1.92-1.88 (m, 1H). MS (ESI+) calculated for $\mathrm{C}_{21} \mathrm{H}_{23} \mathrm{~F}_{3} \mathrm{~N}_{4} \mathrm{O}_{3} \mathrm{~S}$ [M+1]+: 468.5, observed: 469.5. 


\section{Synthesis of 4-(1-((2-morpholino-5-(trifluoromethyl)phenyl)carbamoyl)pyrrolidin-2-}

yl)pyridine-1-ium chloride (14)

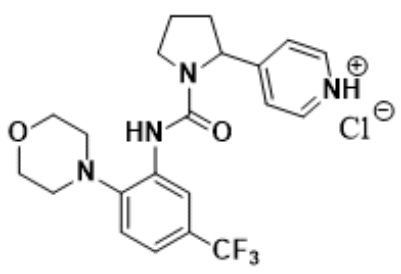

14

$N$-(2-morpholino-5-(truifluoromethyl)phenyl)-2-(pyridine-4-yl)-pyrrolidine-1-carboxamide $(0.052 \mathrm{~g}, 2.0 \mathrm{mmol})$ was stirred in $4 \mathrm{~mL}$ of a $3 \mathrm{~N}$ solution of $\mathrm{HCl}$ in diethyl ether at room temperature overnight. Crude was concentrated in vacuo and lyophilized to obtain pure product as an orange solid in $100 \%$ yield. ${ }^{1} \mathrm{H}$ NMR ( $\left.\mathrm{D}_{2} \mathrm{O}, 400 \mathrm{MHZ}\right): \delta 8.74(\mathrm{~d}, J=4.0 \mathrm{~Hz}, 2 \mathrm{H}), 8.02(\mathrm{~d}, J$ $=8.0 \mathrm{~Hz}, 2 \mathrm{H}), 7.89(\mathrm{~s}, 1 \mathrm{H}), 7.50(\mathrm{~d}, J=4.0 \mathrm{~Hz}, 1 \mathrm{H}), 7.40(\mathrm{~d}, J=8.0 \mathrm{~Hz}, 1 \mathrm{H}), 5.33-5.30(\mathrm{~m}, 1 \mathrm{H})$, 3.97-3.93 (m, 5H), 3.83-3.77 (m, $1 \mathrm{H}), 3.03(\mathrm{~s}, 4 \mathrm{H}), 2.65-2.56(\mathrm{~m}, 1 \mathrm{H}), 2.21-2.16(\mathrm{~m}, 2 \mathrm{H}), 2.12-$ 1.98 (m, 1H). MS (ESI+) calculated for C21H24ClF3N4O2 [M+1]+: 456.9, observed: $421.3-\mathrm{HCl}$ The following compounds were synthesized following general procedure for the synthesis of $\mathbf{1 4}$

4-(1-((2-morpholinophenyl)carbamoyl)pyrrolidin-2-yl)pyridine-1-ium chloride (16)

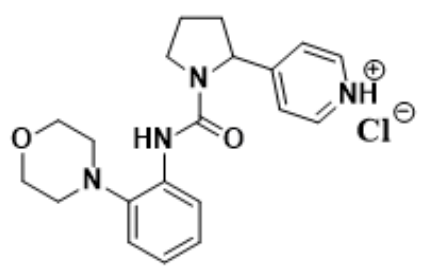


Obtained as an orange solid in $100 \%$ yield. ${ }^{1} \mathrm{H}$ NMR $\left(\mathrm{D}_{2} \mathrm{O}, 400 \mathrm{MHZ}\right): \delta 8.75(\mathrm{~d}, J=4.0 \mathrm{~Hz}, 2 \mathrm{H})$, $8.05(\mathrm{~d}, J=8.0 \mathrm{~Hz}, 2 \mathrm{H}), 7.71(\mathrm{~d}, J=4.0 \mathrm{~Hz}, 1 \mathrm{H}), 7.56-7.54(\mathrm{~m}, 2 \mathrm{H}), 7.52-7.50(\mathrm{~m}, 1 \mathrm{H}), 5.35(\mathrm{~s}$, 1H), 4.11-4.00 (m, 5H), $3.86(\mathrm{q}, J=8.0 \mathrm{~Hz}, 1 \mathrm{H}), 3.68-3.65(\mathrm{~m}, 4 \mathrm{H}), 2.66-2.57(\mathrm{~m}, 1 \mathrm{H}), 2.19-2.15$ $(\mathrm{m}, 2 \mathrm{H}), 2.03-2.00(\mathrm{~m}, 1 \mathrm{H})$. MS (ESI+) calculated for $\mathrm{C} 20 \mathrm{H} 25 \mathrm{ClF} 3 \mathrm{~N} 4 \mathrm{O} 2[\mathrm{M}+1]+: 388.9$, observed: 353.2 - $\mathrm{HCl}$.

\section{$N$-(2-(morpholino-5-(trifluoromethyl)phenyl)-2-(pyridin-4-yl)pyrrolidine-1-carboxamide}

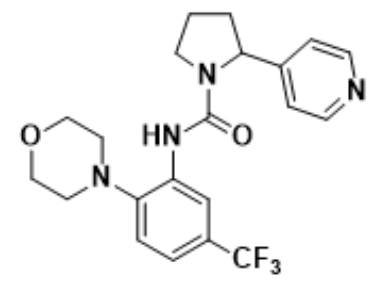

Orange solid. ${ }^{1} \mathrm{H}$ NMR (DMSO-d $\left.6,400 \mathrm{MHZ}\right): \delta 8.52(\mathrm{~d}, J=8.0 \mathrm{~Hz}, 2 \mathrm{H}), 8.48(\mathrm{~s}, 1 \mathrm{H}), 7.93$ (s, br, $1 \mathrm{H}), 7.39(\mathrm{~d}, J=8.0 \mathrm{~Hz}, 1 \mathrm{H}), 7.31(\mathrm{~d}, J=8.0 \mathrm{~Hz}, 2 \mathrm{H}), 7.28(\mathrm{dd}, J=4.0 \mathrm{~Hz}, 2.0 \mathrm{~Hz}, 1 \mathrm{H}), 5.09$ $(\mathrm{d}, J=8.0 \mathrm{~Hz}, 1 \mathrm{H}), 3.82(\mathrm{~s}, 1 \mathrm{H}), 3.67-3.60(\mathrm{~m}, 4 \mathrm{H}), 2.79(\mathrm{~s}, 4 \mathrm{H}), 2.44-2.34(\mathrm{~m}, 1 \mathrm{H}), 1.97(\mathrm{~s}, 1 \mathrm{H})$, 1.82-1.80 (m, 2H). ${ }^{13} \mathrm{C}$ NMR (DMSO-d $\left.6,100 \mathrm{MHz}\right): 152.82,149.61,143.67,135.31,125.64$, $125.45,125.14,122.94,121.67,120.95,118.46,113.87,113.83,66.57,59.39,51.48,46.52$. HRMS (ESI+) calculated for $\mathrm{C}_{21} \mathrm{H}_{23} \mathrm{~F}_{3} \mathrm{~N}_{4} \mathrm{O}_{3} \mathrm{~S}$ [M+1]+: 421.188, observed: 421.188. 


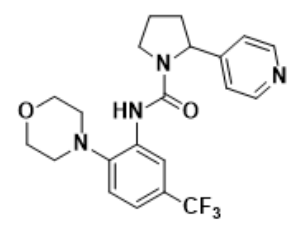

3

M3 DMSO

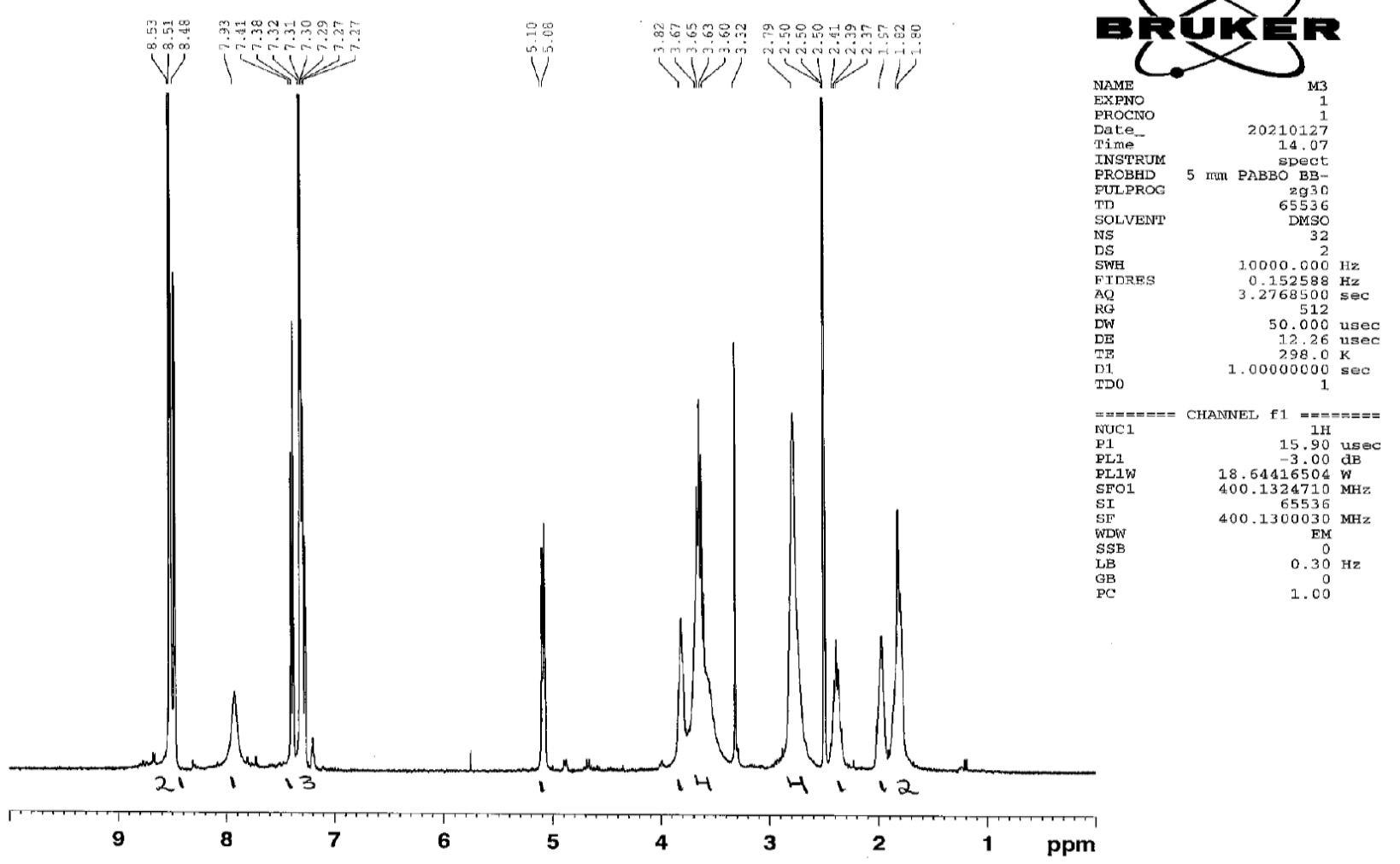



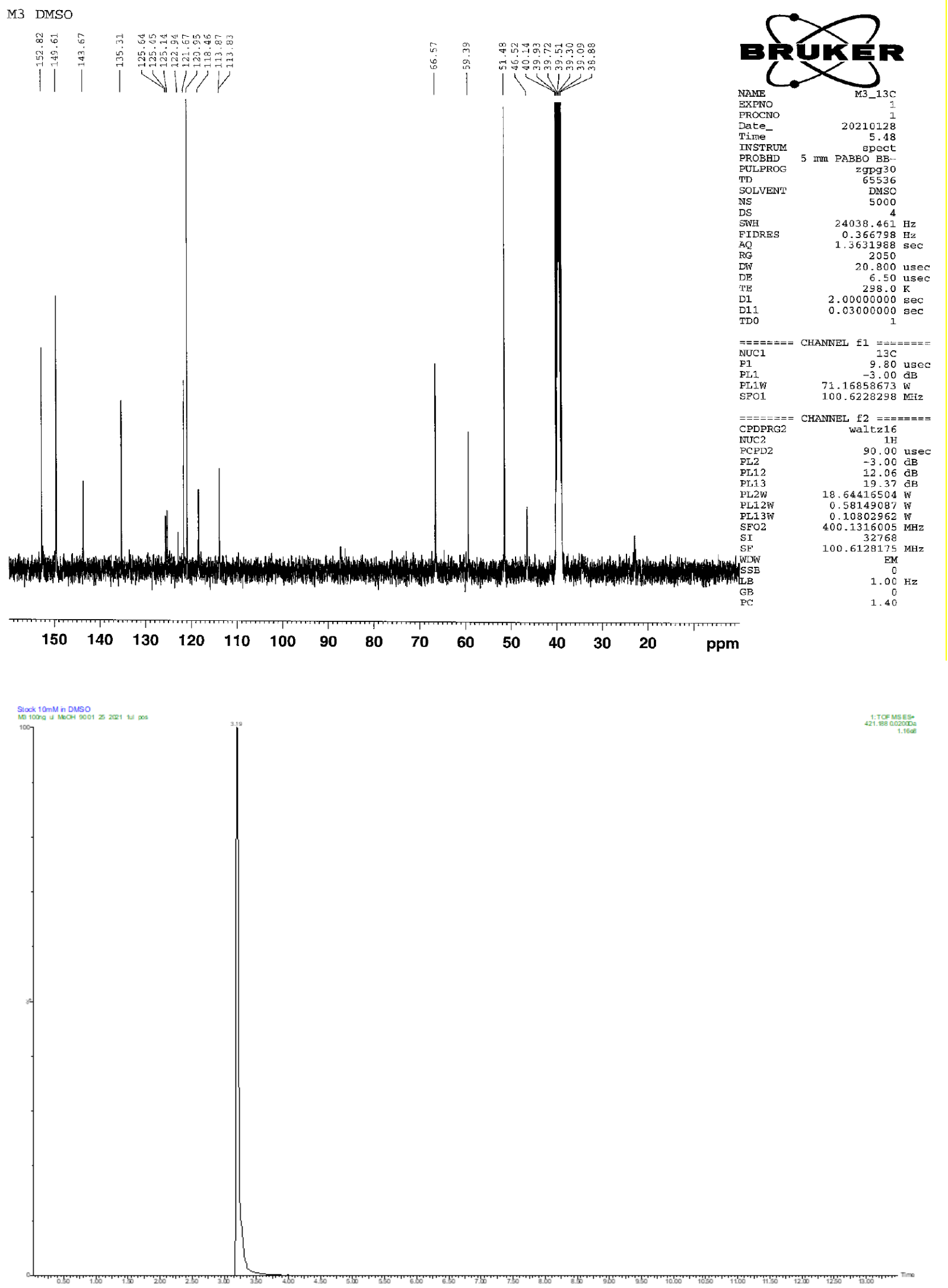
References:

1. Flanigan, T. L.; Owen, C. R.; Gayer, C.; Basson, M. D. Supraphysiologic extracellular pressure inhibits intestinal epithelial wound healing independently of luminal nutrient flow. Am. J. Surg. 2008, 196, 683-689.

2. Owen, C. R.; Yuan, L.; Basson, M. D. Smad3 knockout mice exhibit impaired intestinal mucosal healing. Lab. Invest. 2008, 88, 1101-1109.

3. Compounds $\mathbf{2 4}$ and $\mathbf{2 6}$ were purchase from Enamine Ltd with $>90 \%$ purity.

4. Blasco, V.; Cunat, A. C.; Sanz-Cervera, J. F.; Marco, J. A.; Falomir, E.; Murga, J.; Carda, M. Arylureas derived from colchicine: Enhancement of colchicine oncogene downstream regulation. Eur. J. Med. Chem., 2018, 150, 817-828. 